\title{
The effect of severe and moderate hypoxia on exercise at a fixed level of perceived exertion
}

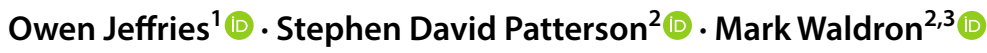

Received: 16 December 2018 / Accepted: 21 February 2019 / Published online: 1 March 2019

(c) The Author(s) 2019

\begin{abstract}
Purpose The purpose of this study was to determine the primary cues regulating perceived effort and exercise performance using a fixed-RPE protocol in severe and moderate hypoxia.

Methods Eight male participants $\left(26 \pm 6\right.$ years, $76.3 \pm 8.6 \mathrm{~kg}, 178.5 \pm 3.6 \mathrm{~cm}, 51.4 \pm 8.0 \mathrm{~mL} \mathrm{~kg}^{-1} \mathrm{~min}^{-1} \dot{V} \mathrm{O}_{2 \max }$ ) completed three exercise trials in environmental conditions of severe hypoxia $\left(\mathrm{F}_{\mathrm{I}} \mathrm{O}_{2} 0.114\right)$, moderate hypoxia $\left(\mathrm{F}_{\mathrm{I}} \mathrm{O}_{2} 0.152\right)$, and normoxia $\left(\mathrm{F}_{\mathrm{I}} \mathrm{O}_{2}\right.$ 0.202). They were instructed to continually adjust their power output to maintain a perceived effort (RPE) of 16 , exercising until power output declined to $80 \%$ of the peak 30 -s power output achieved.

Results Exercise time was reduced (severe hypoxia $428 \pm 210 \mathrm{~s}$; moderate hypoxia $1044 \pm 384$ s; normoxia $1550 \pm$ $590 \mathrm{~s})$ according to a reduction in $\mathrm{F}_{\mathrm{I}} \mathrm{O}_{2}(P<0.05)$. The rate of oxygen desaturation during the first 3 min of exercise was accelerated in severe hypoxia $\left(-5.3 \pm 2.8 \% \mathrm{~min}^{-1}\right)$ relative to moderate hypoxia $\left(-2.5 \pm 1.0 \% \mathrm{~min}^{-1}\right)$ and normoxia $\left(-0.7 \pm 0.3 \% \mathrm{~min}^{-1}\right)$. Muscle tissue oxygenation did not differ between conditions $(P>0.05)$. Minute ventilation increased at a faster rate according to a decrease in $\mathrm{F}_{\mathrm{I}} \mathrm{O}_{2}$ (severe hypoxia 27.6 \pm 6.6 ; moderate hypoxia 21.8 \pm 3.9 ; normoxia 17.3 \pm 3.9 $\left.\mathrm{L} \min ^{-1}\right)$. Moderate-to-strong correlations were identified between breathing frequency $(r=-0.718, P<0.001)$, blood oxygen saturation $(r=0.611, P=0.002)$, and exercise performance.
\end{abstract}

Conclusions The primary cues for determining perceived effort relate to progressive arterial hypoxemia and increases in ventilation.

Keywords Arterial oxygen saturation $\cdot$ Hypoxemia $\cdot$ Cognition $\cdot$ Ventilation $\cdot$ Exercise $\cdot$ Altitude

$\begin{array}{ll}\text { Abbreviations } \\ \text { ANOVA } & \text { Analysis of variance } \\ \mathrm{CNS} & \text { Central nervous system } \\ d & \text { Cohen's } d \\ \mathrm{~F}_{\mathrm{I}} \mathrm{O}_{2} & \text { Fraction of inspired oxygen }\end{array}$

Communicated by Michalis G. Nikolaidis.

Owen Jeffries

owen.jeffries@newcastle.ac.uk

Stephen David Patterson

stephen.patterson@stmarys.ac.uk

Mark Waldron

mark.waldron@stmarys.ac.uk

1 Faculty of Medical Sciences, School of Biomedical Sciences, Newcastle University, Newcastle Upon Tyne NE2 4HH, UK

2 School of Sport, Health and Applied Science, St Mary's University, Twickenham, London TW1 4SX, UK

3 School of Science and Technology, University of New England, Armidale, NSW, Australia

$\begin{array}{ll}\mathrm{H}^{+} & \text {Hydrogen } \\ \mathrm{HHb} & \text { Deoxyhaemoglobin } \\ \mathrm{NIRS} & \text { Near-infrared spectroscopy } \\ \mathrm{O}_{2} \mathrm{Hb} & \text { Arterial oxygen saturation } \\ \eta_{p}{ }^{2} & \text { Partial eta-squared } \\ \mathrm{PETCO}_{2} & \text { End-tidal carbon dioxide } \\ \mathrm{PETO}_{2} & \text { End-tidal oxygen } \\ \mathrm{PNS} & \text { Peripheral nervous system } \\ \mathrm{RPE} & \text { Rating of perceived exertion } \\ \mathrm{SpO}_{2} & \text { Oxygen saturation } \\ \mathrm{tHb} & \text { Total haemoglobin } \\ \mathrm{TSI} & \text { Tissue saturation index } \\ \dot{V} \mathrm{O}_{2} & \text { Oxygen consumption } \\ \dot{V} \mathrm{O}_{2 \max } & \text { Maximal oxygen uptake } \\ \dot{V} \mathrm{CO}_{2} & \text { Expired carbon dioxide }\end{array}$




\section{Introduction}

Exercise performance during an acute exposure to hypoxia is impaired via a reduction in arterial oxygen content (Fulco et al. 1996, 1998; Calbet et al. 2003a; Amann et al. 2006b; Romer et al. 2006). In moderate hypoxia, where the oxygen fraction of inspired air is reduced to $~ 13-15 \%$ $\left(\mathrm{F}_{\mathrm{I}} \mathrm{O}_{2}\right.$ 0.13-0.15), decrements in performance have been attributed to a rise in peripheral markers of muscle fatigue, which generate afferent feedback to down-regulate motor output from the central nervous system (CNS) (Amann et al. 2006b, 2007; Romer et al. 2007). A so-called 'sensory limit' (Gandevia 2001), therefore, restricts the manifestation of peripheral fatigue to prevent catastrophic failure of any one system. In severe hypoxia $\left(\mathrm{F}_{\mathrm{I}} \mathrm{O}_{2}<0.115\right)$, larger reductions in exercise capacity have been described despite relatively less evidence of peripheral fatigue (Amann et al. 2007). Here, a hypoxia-sensitive 'central' component of fatigue mediates a reduction in central motor output via brain hypoxia (Subudhi et al. 2009; Vogiatzis et al. 2011; Millet et al. 2012; Goodall et al. 2012), thus limiting maximal exercise capacity. Indeed, in experiments where $\mathrm{F}_{\mathrm{I}} \mathrm{O}_{2}$ is increased at the point of task failure, exercise performance can be prolonged in severe and moderate hypoxia (Amann et al. 2007; Torres-Peralta et al. 2016).

Central processing of the perception of effort and its role in setting exercise intensity is heavily debated. The subjective rating of perceived exertion, termed RPE, is a psychophysiological concept (Borg 1982; Morgan 1994) that centrally integrates perceptual, peripheral, experiential, and environmental sensory cues (Hampson et al. 2001). Indeed, Borg (1982) described the RPE as a conscious representation of multiple inputs. To further understand how perceived exertion modulates self-regulated exercise, a fixed-RPE protocol was developed, referred to as the RPE clamp (Tucker et al. 2006). Here, participants exercise at a pre-determined fixed level of perceived exertion on the RPE scale (typically 16, < 'very hard') and modulate their workload according to the perceived mismatches between the expected and actual RPE (Tucker 2009). In an eloquent design, a recent study controlled the rate of arterial hypoxemia $\left(\mathrm{SpO}_{2} 98\right.$ to $\left.70 \%\right)$ through manipulations in $\mathrm{F}_{\mathrm{I}} \mathrm{O}_{2}$, demonstrating that the rate of decline in power output was reliant on the rate change of arterial oxygenation during self-regulated exercise performance (Farra et al. 2017). Whilst this provides evidence of the relationship between $\mathrm{SpO}_{2}$ and the perception of exercise intensity in contrived ambient conditions, it overlooks the responses under fixed reductions in $\mathrm{F}_{\mathrm{I}} \mathrm{O}_{2}$, such as that commonly encountered at altitude. This is important, since exposure to steady-state $\mathrm{F}_{\mathrm{I}} \mathrm{O}_{2}$ conditions provides an opportunity for physiological compensations, such as increased muscle oxygen delivery or extraction. Based on their findings, the rate of change in $\mathrm{SpO}_{2}$ under steadystate $\mathrm{F}_{\mathrm{I}} \mathrm{O}_{2}$ is likely to determine exercise perception and tolerance, yet this is not currently known. These collective organ-level changes could feasibly offset the deleterious effects of hypoxia or, more importantly, complicate the afferent feedback process. We hypothesized that, during exercise at a fixed RPE, power output would decrease in accordance with a reduction in $\mathrm{F}_{\mathrm{I}} \mathrm{O}_{2}$. Therefore, we examined exercise performance using a fixed RPE protocol in severe and moderate hypoxia relative to normoxia, with the aim of determining the relationship between time to exhaustion and a combination of acute physiological responses.

\section{Materials and methods}

\section{Participants}

Eight male participants volunteered to take part in this study (mean \pm SD: age $26 \pm 6$ years; body mass $76.3 \pm 8.6 \mathrm{~kg}$; stature $178.5 \pm 3.6 \mathrm{~cm}$; maximal oxygen consumption, $\dot{V}$ $\mathrm{O}_{2 \max } 51.4 \pm 8.0 \mathrm{~mL} \mathrm{~kg}^{-1} \mathrm{~min}^{-1}$ ). All participants were sea-level residents and none had recently travelled to altitude in the 3 months prior to the study. Written informed consent was obtained from each participant. Participants were instructed to avoid consumption of alcohol or caffeinated products for $24 \mathrm{~h}$ before each visit, as well as strenuous exercise $48 \mathrm{~h}$ before testing and to arrive fully hydrated. All participants gave written informed consent. Ethical approval was provided by the St Mary's University ethics committee (ref: SMEC_2017-18_012), which was conducted in accordance with the 1964 Helsinki declaration.

\section{Study design}

A randomized, single-blind, crossover design was adopted to examine the effect of breathing different oxygen fractions of air $\left(\mathrm{F}_{\mathrm{I}} \mathrm{O}_{2}\right)$ on exercise at a fixed level of perceived exertion using an RPE-clamp protocol (Tucker et al. 2006). A-priori sample size was calculated using $\mathrm{G}^{*}$ Power (Version 3.1.9.3). This was determined according to cycling performance in moderate hypoxia $\left(\mathrm{F}_{\mathrm{I}} \mathrm{O}_{2}=0.13\right)$ relative to normoxia $\left(\mathrm{F}_{\mathrm{I}} \mathrm{O}_{2}\right.$ $=0.20$ ) at $\sim 80 \%$ maximum work rate (mean difference 4.5; pooled SD 2.24) (Goodall et al. 2012). Eight participants per group were deemed sufficient to yield a power of 0.82 at $\alpha=0.05$. The three environmental conditions were randomized by block randomization for groups of three participants at a time using online software (Urbaniak and Plous 2015). Participants visited the laboratory on four separate occasions, each separated by 1 week. During visit 1 , participants conducted baseline testing to establish $\dot{V} \mathrm{O}_{2 \max }$ and to 
familiarise with exercising at an RPE of 16 on the 15-grade Borg scale (Borg 1982) in ambient air. During visits 2-4, participants completed the RPE-clamp protocol in variable $\mathrm{F}_{\mathrm{I}} \mathrm{O}_{2}$ (severe hypoxia, $\mathrm{F}_{\mathrm{I}} \mathrm{O}_{2}=0.114$; moderate hypoxia, $\mathrm{F}_{\mathrm{I}} \mathrm{O}_{2}$ $=0.152$; ambient air, $\mathrm{F}_{\mathrm{I}} \mathrm{O}_{2}=0.202$ ).

\section{Experimental procedures}

\section{Preliminary testing and familiarisation}

All cycling exercise was performed on an electronically braked cycle ergometer (Excalibur Sport, Lode, Groningen, The Netherlands). The cycling setup was recorded on the first visit and replicated for subsequent visits. Participants performed a 5-min warm-up at $100 \mathrm{~W}$ and a fixed cadence of $80 \mathrm{r} \mathrm{min}^{-1}$. The incremental ramp test began at $120 \mathrm{~W}$ and workload increased at a rate of $24 \mathrm{~W} \mathrm{~min}{ }^{-1}$ until volitional fatigue. Expired gases were collected and highest average 30 s reported as $\dot{V} \mathrm{O}_{2 \max }$. RPE was measured at the end of each 1-min stage by pointing to a 6-20 RPE scale, which was held by an investigator. Following the incremental ramp test, two familiarisation exercises were conducted, which were used with the intention of calibrating the participant's RPE-based selection of power output in the main trials. The first exercise was replicated before every test and began at $120 \mathrm{~W}$, with participants controlling resistance on the ergometer, whilst being blinded to actual power output. The aim was to achieve an RPE that they perceived as equalling RPE-16 over a period of 3-5 min. The test was stopped when participants indicated that they had reached the desired work intensity. The second test began at $20 \%$ below the power output selected in test 1 and participants were asked to regulate resistance on the ergometer, by manually toggling up or down on the ergometer's controls, to maintain an RPE of 16. Participants were also given significant time to discuss and understand the RPE protocol with the researchers both before and after these initial familiarisation trials.

\section{Fixed-RPE protocol}

Participants performed three randomized experimental trials, separated by 1 week in a hypoxic chamber (Sporting Edge, Basingstoke, UK). For each participant, the experimental trials were conducted at the same time of day to eliminate the effect of circadian variation. Participants performed a 5 -min warm-up at $100 \mathrm{~W}$ and a fixed cadence of $80 \mathrm{r} \mathrm{min}^{-1}$. During experimental testing, the cycling ergometer was in hyperbolic mode, whereby the participant could adjust their power output. No visual feedback was provided, except for cadence. After being fitted with a near-infrared spectroscopy (NIRS) optode, a heart rate chest strap, and facemask, the participants entered the chamber. Participants immediately completed the standardised RPE-ramp protocol to establish setting of RPE over a 3-5-min period. Participants then rested for 5 min to collect baseline data. Finally, they began the fixed-RPE protocol, freely regulating their power output, starting 20\% below their self-selected RPE, as determined in the standard ambient air during their first visit. Participants were instructed to cycle at a power output that was perceived to represent an RPE of 16 on the 15-grade Borg scale (Borg 1982) and to adjust their power output, such that an RPE of 16 was maintained. An RPE of 16 represents a verbal cue of between 'hard' and 'very hard' on the Borg Scale. During the fixed-RPE trial, the highest power output achieved during a 30-s period in each condition was recorded and participants exercised until their power output declined to $80 \%$ of this initial value. The trial was stopped when power output fell below this value for $>10$-s. Verbal feedback was delivered in a standard format to remind participants to maintain an RPE of 16 at 1 min intervals throughout the trial. Participants were encouraged to constantly reassess whether they were still exercising at RPE-16. They were blinded to distance covered, elapsed time, heart rate, and power output.

\section{Environmental chamber}

Oxygen fraction was controlled by an environmental hypoxic chamber (Sporting Edge, Basingstoke, UK). The three conditions were maintained across all trials as follows: severe hypoxia $\left(\mathrm{F}_{\mathrm{I}} \mathrm{O}_{2}=0.114 ; \mathrm{P}_{\mathrm{I}} \mathrm{O}_{2}=82 \pm\right.$ $1 \mathrm{mmHg})$, moderate hypoxia $\left(\mathrm{F}_{\mathrm{I}} \mathrm{O}_{2}=0.152 ; \mathrm{P}_{\mathrm{I}} \mathrm{O}_{2}=109\right.$ $\pm 1 \mathrm{mmHg})$, and ambient air $\left(\mathrm{F}_{\mathrm{I}} \mathrm{O}_{2}=0.202 ; \mathrm{P}_{\mathrm{I}} \mathrm{O}_{2}=144\right.$ $\pm 2 \mathrm{mmHg}$ ). Temperature and humidity were controlled throughout at $19.4 \pm 0.6{ }^{\circ} \mathrm{C}$ and $39 \pm 4.3 \%$, for all sessions and barometric pressure was recorded as $1013 \pm 4 \mathrm{hPA}$.

\section{Cardiorespiratory measures}

Expired gases were measured breath-by-breath to assess oxygen consumption $\left(\dot{V} \mathrm{O}_{2}\right)$, minute ventilation, breathing frequency, tidal volume and end-tidal oxygen $\left(\mathrm{PETO}_{2}\right)$, and carbon dioxide $\left(\mathrm{PETCO}_{2}\right)$ continuously throughout the test (Vyntus CPX; CareFusion; Hochberg, Germany) and averaged into 15 -s epochs across all the trials. The gas analyzer was calibrated before every trial with the gases of known concentration $\left(15.95 \mathrm{O}_{2}, 4.97 \% \mathrm{CO}_{2}, \mathrm{BAL} . \mathrm{N}_{2}\right)$ and the turbine volume transducer was calibrated automatically by the system at flow values of $2 \mathrm{~L} \mathrm{~s}^{-1}$ and 0.2 $\mathrm{L} \mathrm{s}^{-1}$. Heart rate was recorded continuously throughout the trials (Polar Heart Rate Monitor V800, Warwick, UK). $\mathrm{SpO}_{2}$ was sampled at $1 \mathrm{~Hz}$ using a finger pulse oximeter (Vyntus CPX; CareFusion; Hochberg, Germany) attached to the right index finger. 


\section{Near-infrared spectrometry}

Participants were instrumented with an NIRS optode over the right vastus lateralis to monitor the absorption of light in the muscle tissues (Portamon, Artinis Medical Systems, Zetten, The Netherlands). The optode was affixed over the muscle belly of the right vastus lateralis muscle along the vertical axis of the thigh, $2 / 3$ between the greater trochanter and the lateral epicondyle of the femur. The optode was secured with tape and covered with an optically dense cloth to minimize the possibility that extraneous light could influence the NIRS signal. The placement position was marked with indelible ink to ensure accurate placement for future visits. The system is a two-wavelength continuous wave system that simultaneously uses the modified Beer-Lambert law and spatially resolved spectroscopy methods. Changes in tissue oxyhaemoglobin $\left(\mathrm{O}_{2} \mathrm{Hb}\right)$, deoxyhaemoglobin $(\mathrm{HHb})$, and total haemoglobin $(\mathrm{tHb})$ were measured using the differences in the absorption characteristics of infrared light at 760 and $850 \mathrm{~nm}$. Differential path factor (DPF) of 4 was used throughout. NIRS data were connected to a computer by Bluetooth for acquisition at $10 \mathrm{~Hz}$. Tissue oxygenation index (TSI) represents the ratio of $\mathrm{O}_{2} \mathrm{Hb}$-to-tHb concentration and was reported in response to exercise and used to assume changes in intramuscular oxygen status (Ferrari et al. 2004).

\section{Rating of perceived exertion (RPE)}

Participants were thoroughly briefed on the RPE scale before commencing the fixed-RPE trials. In line with the ACSM guidelines (American College of Sports Medicine 2000), participants were instructed to pay close attention to how difficult the exercise felt, combining total exertion, fatigue, and physical stress in hypoxia, without considering one particular factor, such as leg pain, shortness of breath, or anticipation of how they might feel several minutes later. We attempted to anchor the RPE scale by highlighting the self-reported RPE during the early stages of the incremental ramp test (RPE 10-11) and the final stages of the test (RPE 19-20). To further the enable visualisation of the intensity, participants were provided with associations between the RPE and intensity-duration relationships. An example of this was the guidance that an RPE of 13 was akin to a 2-h cycle, whilst holding a conversation; RPE-15 being close to a 1-h steady-state maximal effort, where sustained conversation would be difficult; and RPE-16 being a maximal effort that they could only sustain for around 25-35 min.

\section{Statistical analysis}

All statistical analyses were performed using SPSS (IBM SPSS statistics 22 Inc, USA). A two-way analysis of variance
(ANOVA) for repeated measures was used to test for withingroup effects across time in and conditions. If sphericity was violated a Greenhouse-Geisser correction was applied. When a significant difference was found for a main effect (condition or time), post hoc pairwise comparisons were made, incorporating a Bonferroni adjustment. Magnitude of the effect was calculated with partial eta-squared $\left(\eta_{p}{ }^{2}\right)$ according to the following criteria: 0.02 , small; 0.13 , moderate; 0.26 large, or using Cohen's $d(d)$ for pairwise comparisons using: 0.2, small; 0.5, moderate; 0.8, large (Cohen 1988). Differing trial durations meant that power data were normalized with respect to time. Cardiovascular data were analyzed by averaging 30 -s data at start, middle, and end points, across each trial. Correlations were performed to examine the relationship between the rate change over the first $3 \mathrm{~min}$ of exercise in oxygen saturation, muscle oxygenation, minute ventilation, tidal volume, breathing frequency, oxygen consumption, $\mathrm{PETCO}_{2}$, and heart rate to variance in exercise time in severe hypoxia, moderate hypoxia, and normoxia. Data are presented as mean $\pm \operatorname{SD}(n=8)$. Significance was set at $P<0.05$.

\section{Results}

Upon entering the hypoxic chamber and prior to the main experimental trial, participants conducted a short selfselected ramp to an RPE of 16 over 3-5 min. The achieved power output associated with an RPE of 16 was different between conditions $\left(F_{(1.180,8.262)}=9.558, P=0.012\right.$, $\eta_{p}{ }^{2}=0.577$ ) (Table 1), with pairwise analysis, confirming that the power output achieved in severe hypoxia was reduced relative to moderate hypoxia and normoxia. There were no differences between the peak power achieved in the ramp protocol and the peak power achieved during the experimental trial (Table 1).

\section{Exercise in hypoxia}

During the exercise trial at a fixed RPE of 16, peak power output was achieved between 10 and $20 \%$ of the trial

Table 1 Peak power output during a short ramp to RPE 16 and the peak power achieved during the main experimental exercise trial

\begin{tabular}{llll}
\hline & Severe hypoxia & Moderate hypoxia & Normoxia \\
\hline $\begin{array}{l}\text { Peak power ramp } \\
\text { (W) }\end{array}$ & $170 \pm 30^{* \#}$ & $204 \pm 44$ & $207 \pm 35$ \\
Peak power trial (W) & $183 \pm 29 * \#$ & $201 \pm 35$ & $208 \pm 25$ \\
\hline Values are means \pm SD for eight participants & \\
$* P<0.05$ relative to moderate hypoxia & \\
${ }^{*} P<0.05$, relative to normoxia &
\end{tabular}




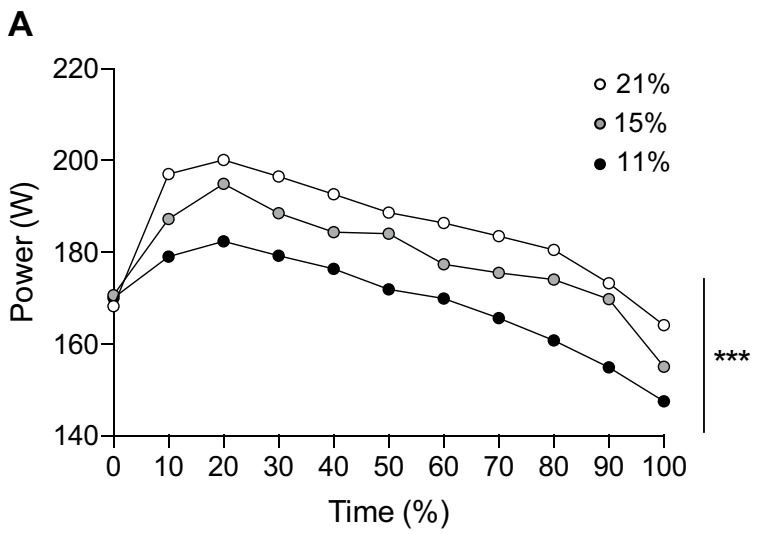

Fig. 1 Power output during exercise at a fixed RPE in variable inspired oxygen fractions. a Average power output relative to exercise time expressed as $100 \%$ of the performance trial in severe hypoxia (black circle), moderate hypoxia (grey circle), and normoxia (white circle). SD has not been included for clarity. b Inter-individual and

duration (Fig. 1a). Exercise time was reduced in accordance with a reduction in $\mathrm{F}_{\mathrm{I}} \mathrm{O}_{2}$ (severe hypoxia $=428 \pm 210 \mathrm{~s}$; moderate hypoxia $=1044 \pm 384 \mathrm{~s} ;$ normoxia $=1550 \pm 590 \mathrm{~s})$ $\left(F_{(2,14)}=24.526, P<0.001, \eta_{p}{ }^{2}=0.778\right)$ (Fig. 1b). Modulation of power output across the exercise trial decreased with time $\left(F_{(10,70)}=32.950, P=0.000, \eta_{p}{ }^{2}=0.825\right)$ and was different between condition $\left(F_{(1.555,15.55)}=60.432, P=0.000\right.$, $\eta_{p}{ }^{2}=0.858$ ) (Fig. 1a). However, the rate of decrease in power output identified from $20 \%$ into the trial until exercise cessation $(100 \%)$ was not significantly different between conditions $(P<0.05)$.

\section{Blood oxygen saturation}

At baseline, $\mathrm{SpO}_{2}$ in the $30 \mathrm{~s}$ prior to exercise was reduced in both severe $(85 \pm 2.7 \%)$ and moderate hypoxia $(94 \pm 2.0 \%)$ relative to normoxia $(98 \pm 0.7 \%)$

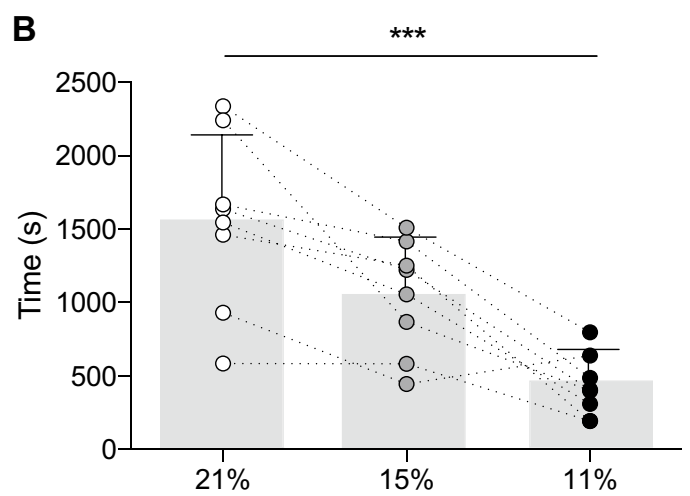

mean performance time across the three trials at a fixed RPE, exercise was terminated when power output dropped $20 \%$ below the peak power output achieved in the trial. All data are mean \pm SD. $* * *$ Significant main effect of condition

$\left(F_{(2,14)}=132.501, P<0.0001, \eta_{p}{ }^{2}=0.950\right)$. During exercise, $\mathrm{SpO}_{2}$ decreased with time $\left(F_{(2,14)}=55.871\right.$, $\left.P<0.0001, \eta_{p}^{2}=0.889\right)$ and decreased relative to $\mathrm{F}_{\mathrm{I}} \mathrm{O}_{2}$ $\left(F_{(2,14)}=197.899, P<0.0001, \eta_{p}{ }^{2}=0.966\right)$, demonstrating an interaction effect $\left(F_{(4,28)}=18.255, P<0.0001\right.$, $\left.\eta_{p}{ }^{2}=0.723\right)$. Follow-up pairwise analysis confirmed that all conditions were different from each other $(P<0.05)$. End-exercise levels were decreased in severe hypoxia $(72 \pm 5 \%)$ and moderate hypoxia $(87 \pm 3 \%)$ relative to normoxia $(96 \pm 2 \%)$. The rate of oxygen desaturation the first $3 \mathrm{~min}$ of exercise was accelerated in severe hypoxia $\left(-5.3 \pm 2.8 \% \mathrm{~min}^{-1}\right)$ relative to moderate hypoxia $\left(-2.5 \pm 1.0 \% \mathrm{~min}^{-1}\right)$ with both being faster than normoxia where the change was small $\left(-0.7 \pm 0.3 \% \mathrm{~min}^{-1}\right)$ $\left(F_{(2,14)}=18.571, P<0.0001, \eta_{p}{ }^{2}=0.726\right)$ (Table 1). At the point of exercise cessation relative to baseline measures, $\mathrm{SpO}_{2}$ was decreased by $\sim 14 \%$ in severe hypoxia and $~$

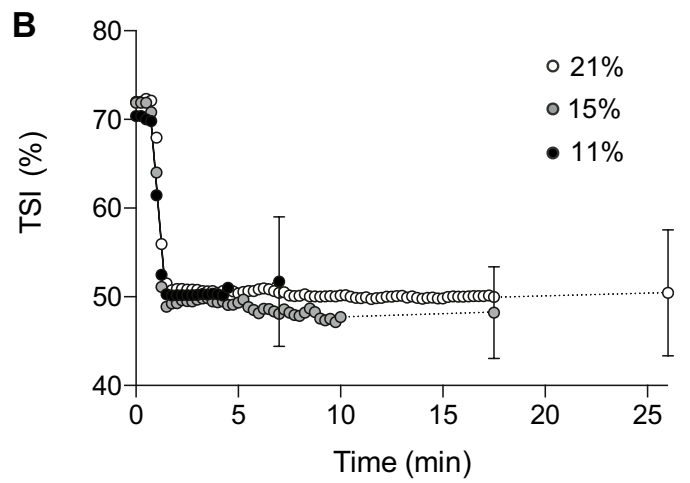

Each condition is represented as severe hypoxia (black circle), moderate hypoxia (grey circle), and normoxia (white circle)
Fig. 2 Blood oxygen saturation and vastus lateralis tissue oxygen saturation at a fixed RPE in variable inspired oxygen fractions. a Oxygen saturation $\left(\mathrm{SpO}_{2}\right)$; b vastus lateralis tissue saturation index (TSI).

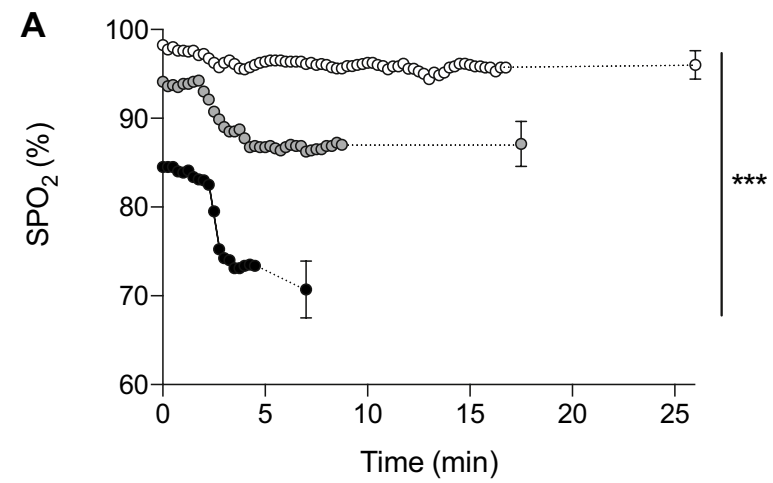


$8 \%$ in moderate hypoxia and maintained within $\sim 3 \%$ in normoxia (Fig. 2a).

\section{Muscle oxygenation}

Muscle oxygenation at rest was similar across all the conditions (severe hypoxia, $70 \pm 3 \% \mathrm{TSI}$; moderate hypoxia, 72 $\pm 3 \% \mathrm{TSI}$; normoxia, $72 \pm 3 \% \mathrm{TSI}$ ). TSI decreased with time $\left(F_{(1.122,7.855)}=122.771, P=0.000, \eta_{p}{ }^{2}=0.946\right)$ falling rapidly during the first few seconds of exercise in each $\mathrm{F}_{\mathrm{I}} \mathrm{O}_{2}$ and reaching a steady state that was not different between each condition $\left(F_{(2,14)}=0.906, P=0.426, \eta_{p}{ }^{2}=0.115\right)$. Indeed, the rate of change in muscle oxygenation in each $\mathrm{F}_{\mathrm{I}} \mathrm{O}_{2}$ was not different $(P<0.05)$. At the point of withdrawal from the task, TSI was remarkably similar between conditions (severe hypoxia, $52 \pm 7 \% \mathrm{TSI}$; moderate hypoxia, $48 \pm 5 \% \mathrm{TSI}$; normoxia, $51 \pm 6 \%$ TSI) (Fig. 2b).

\section{Ventilatory measures}

Minute ventilation increased with time $\left(F_{(1.107,7.750)}=233.333, P<0.0001, \eta_{p}{ }^{2}=0.971\right)$ and was different between $\mathrm{F}_{\mathrm{I}} \mathrm{O}_{2}\left(F_{(2,14)}=25.166, P=0.001\right.$, $\left.\eta_{p}{ }^{2}=0.782\right)$ with an interaction effect $\left(F_{(4,28)}=5.767\right.$, $\left.P=0.002, \eta_{p}{ }^{2}=0.452\right)$. Follow-up pairwise analysis confirmed that minute ventilation across the entire trial was increased in severe hypoxia $(P=0.002, d=1.83)$ and moderate hypoxia $(P<0.001, d=1.51)$ relative to normoxia (Fig. 3a). The rate at which minute ventilation increased over the first $3 \mathrm{~min}$ of exercise was accelerated relative to reducing $\mathrm{F}_{\mathrm{I}} \mathrm{O}_{2}\left(F_{(2,14)}=22.868, P<0.001, \eta_{p}{ }^{2}=0.766\right)$, with severe hypoxia $\left(27.6 \pm 6.6 \mathrm{~L} \mathrm{~min}^{-1}\right)$ and moderate hypoxia $\left(21.8 \pm 3.9 \mathrm{~L} \mathrm{~min}^{-1}\right)$ showing faster changes than in normoxia $\left(17.3 \pm 3.9 \mathrm{~L} \mathrm{~min}^{-1}\right)$. To understand the changes in minute ventilation, breathing frequency and tidal volume were further analyzed. Breathing frequency increased with time $\left(F_{(2,14)}=128.123, P<0.0001, \eta_{p}{ }^{2}=0.948\right)$, but was not different between condition $(P>0.05)$, but showed an interaction effect $\left(F_{(4,28)}=45.859, P=0.005, \eta_{p}{ }^{2}=0.405\right)$ (Fig. 3c). The rate of increase across the first 3 min of exercise was different in each $\mathrm{F}_{\mathrm{I}} \mathrm{O}_{2}\left(F_{(2,14)}=25.458, P<0.001\right.$, $\eta_{p}{ }^{2}=0.784$ ) with breathing frequency increasing by $4.4 \pm 1.9$ breaths $\min ^{-1}$ in severe hypoxia $(P=0.008, d=-1.45)$ and $3.5 \pm 1.4$ breaths $\min ^{-1}$ in moderate hypoxia $(P<0.001, d$ $=-1.16)$ relative to normoxia $\left(1.9 \pm 1.5\right.$ breaths $\left.\mathrm{min}^{-1}\right)$. Tidal volume increased with time $\left(F_{(2,14)}=12.798\right.$, $\left.P<0.001, \eta_{p}{ }^{2}=0.921\right)$ and condition $\left(F_{(2,14)}=23.586\right.$, $\left.P<0.001, \eta_{p}{ }^{2}=0.771\right)$. Follow-up pairwise analysis confirmed tidal volume was increased in severe hypoxia $(P=0.002, d=1.65)$ and moderate hypoxia $(P=0.014$.
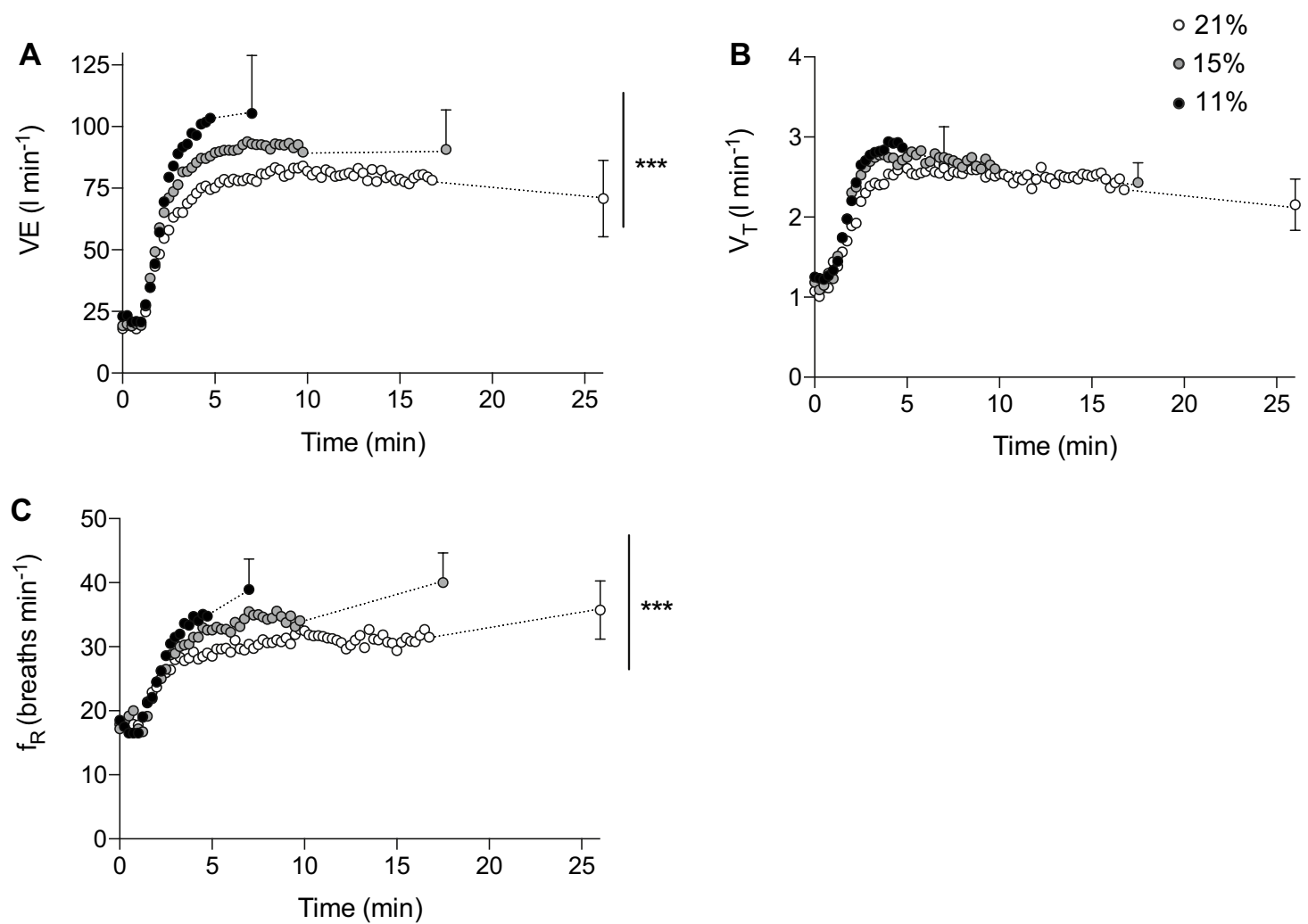

Fig. 3 Breathing parameters at a fixed RPE in variable inspired oxygen fractions. a Minute ventilation, ( $\dot{V}$ E); b tidal volume (VT); c breathing frequency $(f R)$. Each condition is represented as severe hypoxia (black circle), moderate hypoxia (grey circle), and normoxia (white circle) 
Table 2 Rate of change in physiological variables during the first $3 \mathrm{~min}$ of exercise in severe hypoxia, moderate hypoxia, and normoxia

\begin{tabular}{|c|c|c|c|}
\hline & Severe hypoxia & Moderate hypoxia & Normoxia \\
\hline Time (s) & $428 \pm 210^{* \#}$ & $1044 \pm 384 *$ & $1550 \pm 590$ \\
\hline Power $\left(\mathrm{W} \% \mathrm{~min}^{-1}\right)$ & $-0.4 \pm 0.1$ & $-0.3 \pm 0.1$ & $-0.4 \pm 0.1$ \\
\hline $\mathrm{SpO}_{2}\left(\% \min ^{-1}\right)$ & $-5.3 \pm 2.8^{* \#}$ & $-2.5 \pm 1.0^{*}$ & $-0.7 \pm 0.3$ \\
\hline $\operatorname{TSI}\left(\% \min ^{-1}\right)$ & $-3.1 \pm 0.7$ & $-3.4 \pm 1.2$ & $-3.6 \pm 1.5$ \\
\hline Heart rate $\left(\mathrm{bpm} \mathrm{min}^{-1}\right)$ & $23.6 \pm 4.7$ & $22.0 \pm 6.5$ & $22.4 \pm 2.2$ \\
\hline Minute ventilation $\left(\mathrm{L} \mathrm{min}^{-1}\right)$ & $27.6 \pm 6.6^{* \#}$ & $21.8 \pm 3.9^{*}$ & $17.3 \pm 3.9$ \\
\hline Breathing frequency (breaths $\min ^{-1}$ ) & $4.4 \pm 1.9 * \#$ & $3.5 \pm 1.4^{*}$ & $1.9 \pm 1.5$ \\
\hline Tidal volume $\left(\mathrm{L} \mathrm{min}^{-1}\right)$ & $0.5 \pm 0.1$ & $0.6 \pm 0.2$ & $0.6 \pm 0.2$ \\
\hline$\dot{V} \mathrm{O}_{2}\left(\mathrm{~L} \mathrm{~min}^{-1}\right)$ & $0.6 \pm 0.1 *$ & $0.8 \pm 0.1 *$ & $0.7 \pm 0.1$ \\
\hline
\end{tabular}

Values are means \pm SD for eight participants

$\mathrm{SpO}_{2}$ oxygen saturation, $\mathrm{TSI}$ tissue saturation index, $\dot{\mathrm{V}} \mathrm{O}_{2}$ oxygen consumption

${ }^{*} P<0.05$ relative to normoxia; ${ }^{\#} P<0.05$, relative to moderate hypoxia $d=1.03$ ) relative to normoxia (Fig. 3b). However, the rate of change in tidal volume between condition was not different $(P<0.05)$ (Table 2). There were main effects of time for $\operatorname{PETCO}_{2}\left(F_{(2,14)}=3.725, P=0.05, \eta_{p}^{2}=0.347\right)$ and condition $\left(F_{(2,14)}=9.768, P=0.002, \eta_{p}{ }^{2}=0.583\right)$, with an interaction effect $\left(F_{(4,28)}=4.258, P=0.008, \eta_{p}{ }^{2}=0.378\right)$. Pairwise comparisons confirmed that $\mathrm{PETCO}_{2}$ was reduced in severe hypoxia relative to normoxia $(P=0.015, d=$ -0.98) (Fig. 4a). There was no effect of time for PETO $_{2}$ $\left(F_{(2,14)}=1.267, P=0.312, \eta_{p}{ }^{2}=0.153\right)$, but a main effect of condition $\left(F_{(2,14)}=1210.515, P<0.001, \eta_{p}{ }^{2}=0.994\right)$, with an interaction effect $\left(F_{(2.014,14.096)}=4.129, P=0.039\right.$, $\left.\eta_{p}{ }^{2}=0.371\right)$. Pairwise comparisons confirmed that $\mathrm{PETO}_{2}$ was reduced in severe hypoxia relative to normoxia $(P<0.001, d=-17.75)$ and moderate hypoxia $(P=0.002$, $d=-17.82$ ) (Fig. 4b).

\section{Cardiorespiratory measures}

There were main effects of time for $\dot{V} \mathrm{O}_{2}$ $\left(F_{(1.073,7.510)}=467.663, P<0.0001, \eta_{p}{ }^{2}=0.985\right)$ and

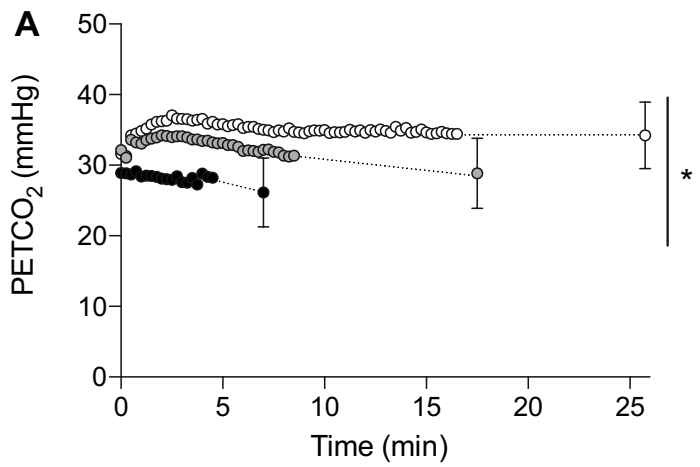

Fig. 4 End-tidal expired air partial pressures during exercise at a fixed RPE in variable inspired oxygen fractions. a End-tidal carbon dioxide $\left(\mathrm{PETCO}_{2}\right)$; b end-tidal oxygen $\left(\mathrm{PETO}_{2}\right)$. Each condition is condition $\left(F_{(2,14)}=35.680, P<0.001, \eta_{p}{ }^{2}=0.836\right)$, with an interaction effect $\left(F_{(4,28)}=15.531, P=0.002\right.$, $\left.\eta_{p}{ }^{2}=0.689\right)$. Pairwise comparisons confirmed that $\dot{V} \mathrm{O}_{2}$ was reduced in severe hypoxia relative to both moderate hypoxia $(P<0.001, d=-1.52)$ and normoxia $(P=0.001$, $d=-1.32$ ) (Fig. 5a). The rate of change in $\dot{V} \mathrm{O}_{2}$ from baseline to 3 min into exercise was different according to $\mathrm{F}_{\mathrm{I}} \mathrm{O}_{2}$ $\left(F_{(2,14)}=8.800, P=0.003, \eta_{p}{ }^{2}=0.557\right)$ with a slower rate change in severe hypoxia $\left(0.6 \pm 0.1 \mathrm{~L} \mathrm{~min}^{-1}\right)$ relative to moderate hypoxia $\left(0.8 \pm 0.1 \mathrm{~L} \mathrm{~min}^{-1}\right)(P=0.024, d=1.42)$ and normoxia $\left(0.7 \pm 0.1 \mathrm{~L} \mathrm{~min}^{-1}\right)(P=0.015, d=1.05)$. $\dot{V} \mathrm{CO}_{2}$ showed main effects for time $\left(F_{(2,14)}=281.038\right.$, $\left.P<0.001, \eta_{p}{ }^{2}=0.976\right)$, but no differences between condition or rate of change $(P>0.05)$. Heart rate increased with time during exercise $\left(F_{(2,14)}=806.597, P<0.001\right.$, $\left.\eta_{p}{ }^{2}=0.991\right)$ and plateaued at a similar point, with no differences between conditions $(P>0.05)$. Descriptively, heart rate appeared to increase and reach steady state faster in severe hypoxia (Fig. 5b); however, there was no significant change in rate during the first $3 \mathrm{~min}$ of exercise between conditions $(P>0.05)$.

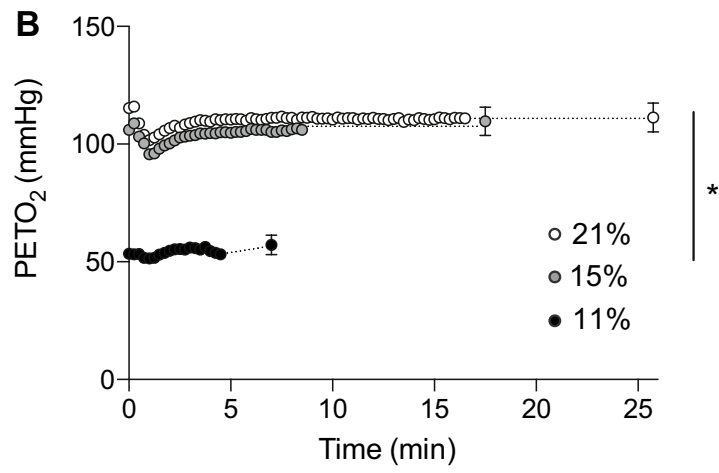

represented as severe hypoxia (black circle), moderate hypoxia (grey circle), and normoxia (white circle) 


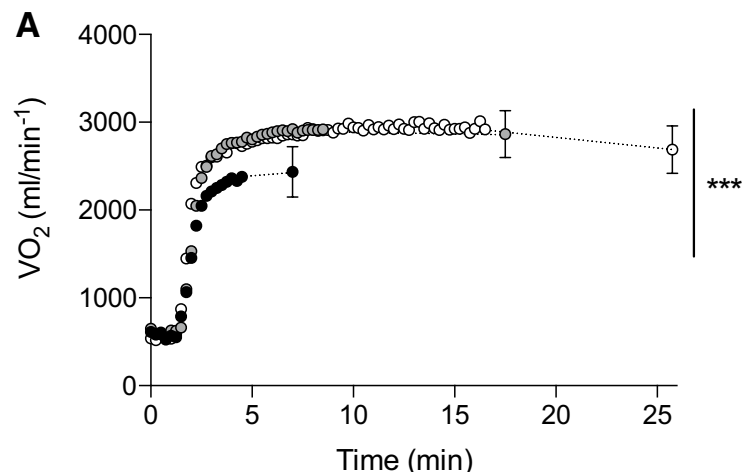

Fig. 5 Oxygen consumption and heart rate responses during exercise at a fixed RPE in variable inspired oxygen fractions. a Oxygen consumption $\left(\dot{V} \mathrm{O}_{2}\right)$; b heart rate (HR). Each condition is represented

\section{Rate change effects on exercise performance}

Moderate-to-strong correlations were identifed between the rate of change during the first $3 \mathrm{~min}$ of exercise in breathing frequency $(r=-0.718, P<0.001)$ and $\mathrm{S}_{\mathrm{p}} \mathrm{O}_{2}(r=0.611$, $P=0.002)$ and exercise duration at a fixed RPE in differing $\mathrm{F}_{\mathrm{I}} \mathrm{O}_{2}$.

\section{Discussion}

The purpose of the study was to investigate the effect of severe and moderate hypoxia on exercise performed at a fixed RPE in reference to normoxia. As anticipated, our findings demonstrate that performance time was diminished when exposed to decreasing $\mathrm{F}_{\mathrm{I}} \mathrm{O}_{2}$, meaning that participants down-regulated their work load as a result of increasing levels of hypoxia. Increases in breathing frequency and blood oxygen desaturation during the early stages of exercise were correlated with reductions in task performance. Despite these changes, oxygen extraction at the muscle (as indicated by NIRS) appeared to be tightly regulated to match the metabolic demand, suggesting that muscle oxygenation is not involved in determining perception during the early stages of setting exercise intensity. Together, the early rate of change in ventilation and arterial hypoxemia appears to drive the selection of exercise intensity associated with a fixed RPE in hypoxia.

Reductions in exercise performance in hypoxia have been attributed to depleted arterial oxygen content (Fulco et al. 1996, 1998; Calbet et al. 2003a; Amann et al. 2006b; Romer et al. 2006). Breathing hypoxic gas leads to a decrease in the arterial partial pressure of oxygen, oxygen saturation of haemoglobin, and the amount of oxygen dissolved in the plasma. Consequently, arterial oxygen content is reduced. Here, a reduction in $\mathrm{F}_{\mathrm{I}} \mathrm{O}_{2}$ decreased exercise time by $~ 72 \%$

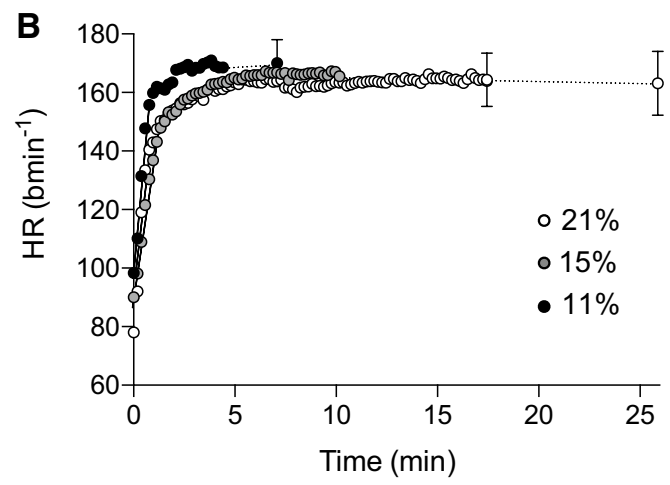

as severe hypoxia (black circle), moderate hypoxia (grey circle), and normoxia (white circle)

in severe hypoxia $\left(\mathrm{F}_{\mathrm{I}} \mathrm{O}_{2}<0.115\right)$ and by $\sim 33 \%$ in moderate hypoxia $\left(\mathrm{F}_{\mathrm{I}} \mathrm{O}_{2} \sim 0.15\right)$, relative to normoxia. There was some evidence of inter-individual responses with two participants showing a reduced sensitivity to hypoxia. Whilst a number factors have been presented to explain responders and non-responders to hypoxia and altitude (Fulco et al. 1998), these individuals performed relatively poorly in normoxia and recorded low aerobic capacity, suggesting that this may be related to fitness status. Across the group, $\mathrm{SpO}_{2}$ was maintained within $3 \%$ of resting levels during normoxia; however, upon acute exposure to moderate hypoxia, resting $\mathrm{S}_{\mathrm{P}} \mathrm{O}_{2}$ was reduced by $\sim 4 \%$ and decreased by a further $8 \%$ across the exercise trial. In severe hypoxia, these reductions in $\mathrm{SpO}_{2}$ were much greater, decreasing by $\sim 14 \%$ at rest, with a further decrease of $14 \%\left(\mathrm{~S}_{\mathrm{P}} \mathrm{O}_{2} \sim 72 \%\right)$ observed at end-exercise. Reductions in exercise performance in moderate hypoxia have largely been attributed to peripheral mechanisms, where a decrease in arterial oxygen content and impaired oxygen delivery to the working muscle leads to a subsequent metabolic perturbation (Hogan et al. 1999). This work has been advanced by studies describing comparable levels of peripheral muscle fatigue via evoked maximal contractions following exhaustive exercise in normoxia and hypoxia despite a substantial reduction in exercise time (Amann et al. 2006b; Romer et al. 2006, 2007; Goodall et al. 2012). Greater reductions in performance described in severe hypoxia have been attributed to greater impairments in pulmonary gas exchange, reduced limb blood flow, and reductions in cardiac output (Calbet et al. 2003a). However, experiments demonstrate rapid improvements in exercise performance and cerebral oxygenation following a fast transition from breathing gas that is severely hypoxic to hyperoxic, at the point of exhaustion, supporting a central role (Amann et al. 2006a).

We examined how the initial exposure to a hypoxic environment would impact determination of the exercise 
intensity associated with 16 on the Borg RPE scale (Borg 1982). Changes in perceived exertion may be determined during the beginning stages of exercise via a range of perceptual, peripheral, experiential, and environmental sensory cues to enable task completion within the physiological limits of the body (Hampson et al. 2001; St Clair Gibson et al. 2006). In both normoxic and moderate hypoxic conditions, the selected power output achieved within several minutes was comparable; however, in severe hypoxia, power output was reduced by $\sim 18 \%$. Whilst the ability to generate maximal power is unaffected by severe levels of hypoxia (Calbet et al. 2003b), supporting the argument that motor drive is unaffected upon acute exposure, the heightened perception of effort observed here may reflect a detrimental effect on decision-making processes (Niedermeier et al. 2017), cognition (McMorris et al. 2017), or a teleoanticipatory reduction in power output to maintain homeostasis (St Clair Gibson et al. 2006). During exercise in hypoxia, cerebral vascular conductance is continually adjusted to maintain oxygen delivery when a reduction in arterial oxygen concentration occurs (Curtelin et al. 2018). However, reductions in cerebral oxygenation have been described at rest and during exercise in hypoxia (Subudhi et al. 2009). Whilst the brain can compensate by increasing oxygen extraction (GonzalezAlonso et al. 2004), neuronal function can also be inhibited (Neubauer et al. 1990) which may impact higher cognitive functions. Whilst we did not directly measure cerebral oxygenation or cerebral blood flow, we did observe a reduction end-tidal carbon dioxide $\left(\mathrm{PETCO}_{2}\right)$ in severe hypoxia relative to normoxia. A close relationship exists between $\mathrm{PETCO}_{2}$ and cerebral blood flow (Ide et al. 2003), suggesting that cerebral blood flow may have been reduced during exercise in severe hypoxia. Hypocapnia reduces cerebral blood flow by as much as $\sim 3 \%$ for every $\sim 1 \mathrm{mmHg}$ change in $\mathrm{PETCO}_{2}$ (Ringelstein et al. 1992). Based on an observed $\sim 8 \mathrm{mmHg}$ difference during exercise in normoxia and severe hypoxia (Fig. 4a), this could equate to a $24 \%$ reduction in cerebral blood flow. It should also be noted that $\mathrm{PETCO}_{2}$ sensitivity is increased by acute exposure to hypoxia (Jensen et al. 1996; Poulin et al. 2002) and the effect on $\mathrm{PETCO}_{2}$ sensitivity has shown differential effects (Fortune et al. 1992; Vovk et al. 2002). Therefore, these observations should be further explored.

At submaximal exercise intensities when arterial oxygenation is reduced, it is probable that oxygen delivery to exercising muscles is compromised. However, compensatory mechanisms increase oxygen extraction or blood flow to maintain muscle oxygen supply (Amann and Calbet 2008). Remarkably, vastus lateralis oxygen saturation assessed via NIRS, did not differ, irrespective of the level of arterial hypoxemia experienced, suggesting comparable oxygenation in the primary exercising muscles during cycling. This has been previously observed during submaximal exercise in both severe and moderate hypoxia (Millet et al. 2012). Here, it was reasoned that afferent signalling, emanating from the metabolic environment at the muscle, is unlikely to have changed between conditions and, therefore, could not explain the reductions in exercise performance. On this basis, it was postulated that tissue deoxygenation was unlikely to play a role in determining exercise intensity. However, the metabolic adaptations and changes in blood flow to maintain constant tissue oxygenation may have contributed to afferent signalling. In the current study, the rate of change in TSI\% during the first 3 min of exercise did not differ according to $\mathrm{F}_{\mathrm{I}} \mathrm{O}_{2}$, yet it transitioned to a lower steady state from the onset of exercise across all the conditions. Therefore, working muscles were able to match oxygen delivery and extraction to meet the metabolic demand during submaximal exercise, despite an increasing physiological perturbation.

Reductions in $\mathrm{F}_{\mathrm{I}} \mathrm{O}_{2}$ also corresponded with increases in minute ventilation. End-exercise minute ventilation was augmented by $41 \%$ and $24 \%$ in severe and moderate hypoxia, respectively, compared to normoxia. Ventilation during exercise is controlled via a balance of centrally mediated feedforward commands and peripheral feedback that increases the rate and depth of breathing according to the demands imposed by the exercise intensity (Kaufman and Forster 1996). Changes in the partial pressure of blood gases are sensed by both central and peripheral chemoreceptors. In the brain, chemoreceptors respond to changes in brain tissue $\mathrm{CO}_{2} /\left[\mathrm{H}^{+}\right]$(Nattie and Li 2009; Tipton et al. 2017). Peripherally, chemoreceptors located in the carotid artery respond to low arterial $\mathrm{O}_{2}$ and high arterial $\mathrm{CO}_{2}$. The increased ventilatory response during the majority of the exercise trial was achieved by an increased tidal volume, which is typically the most efficient mechanical way to increase minute ventilation commensurate with metabolic needs and decreasing arterial blood gas tensions (Tipton et al. 2017). During the early stages of exercise, the rate of increase in breathing frequency was inversely related to the reductions in ambient $\mathrm{F}_{\mathrm{I}} \mathrm{O}_{2}$ and was the strongest predictor of exercise time in hypoxia. The sensations of ventilation and breathing discomfort are consciously monitored during exercise (Robertson 1982). Indeed, the relationship between RPE and ventilation is well established (Cafarelli and Noble 1976; Robertson 1982; Killian 1998; Nicolo et al. 2016). In hypoxia, it is, therefore, possible that the rapid change in ventilation during exercise may have potentiated a greater conscious awareness, contributing to both the initial setting of exercise intensity and the modulation of perceived exercise intensity thereafter.

In an attempt to further examine the acute effects of hypoxia, we determined the rate change of the physiological measures taken during the first 3 min of exercise when an approximate steady state was obtained. We found that rate changes in breathing frequency and blood oxygen 
desaturation showed moderate-to-strong correlations with performance time. A recent study by Farra et al. (2017) elegantly demonstrated that, when the rate of $\mathrm{SpO}_{2}$ was altered via $\mathrm{F}_{\mathrm{I}} \mathrm{O}_{2}$, faster arterial deoxygenation resulted in a greater decline in perceptually controlled exercise performance (Farra et al. 2017). They suggested that RPE was sensitive to both the rate of change and absolute magnitude of arterial deoxygenation, which we can partially support based on a fixed hypoxic environment. Interestingly, in contrast to our findings, they reported no difference in breathing frequency between the experimental conditions (fast, medium and slow desaturations). This may reflect the gradual reduction in $\mathrm{S}_{\mathrm{P}} \mathrm{O}_{2}$ controlled by Farra et al. (2017), which may have masked the relative contributions of the other candidate sensory cues, such as breathing rate, that combine to inform higher brain centres of the homeostatic disturbances. Indeed, these early cues appear to influence the selection of the initial exercise intensities and the subsequent power output that is sustainable for the entire exercise trial. Future work should explore the relationship between breathing frequency and exercise time in hypoxia and practical solutions to reduce breathing frequency may facilitate improvements to performance.

The relationship between exercise regulation, pacing, and RPE is still heavily debated. A three-dimensional framework has recently been proposed as a multidimensional model of volitional self-regulatory control and perceived fatigability (Venhorst et al. 2018). The model combines a sensory-discriminatory dimension (peripheral and central sensations), an affective-motivational dimension (arousal and motivation), and a cognitive-evaluative dimension (exertion and task aversion) (Venhorst et al. 2018). Importantly, this model accounts for both external and internal mediating factors in the generation of RPE. These multiple inputs are, therefore, continually processed, integrated, and interpreted, consciously or otherwise, to alter pacing behaviour in anticipation of potential threats to homeostasis (Hampson et al. 2001; Noakes 2004; Tucker 2009; Venhorst et al. 2018). Such complex psychophysiological interactions, therefore, provide a construct for the observed behavioural differences in pacing in severe and moderate hypoxia when exercising according to a fixed RPE. As we reported, the rate of decrease in power output did not differ between conditions once peak power was achieved; hence, the early setting of an acceptable perceived exercise intensity appears crucial to exercise performance. Whilst it is likely that the exercising template is updated as exercise ensues (Brick et al. 2016), the interplay between such dimensions in generating RPE when challenged with reduced $\mathrm{F}_{\mathrm{I}} \mathrm{O}_{2}$ will require further investigation. Therefore, we propose that the early setting of task intensity in a hypoxic environment is chiefly based upon two primary physiological cues of ventilation and $\mathrm{SpO}_{2}$, thus determining performance.

\section{Limitations}

Attenuated perceptual responses after hypoxic training have been described (Brocherie et al. 2017), suggesting an improved tolerance or acclimation to hypoxia after only one session. The subsequent effects of prior exposure on perception and exercise intensity using the fixed-RPE protocol are unknown and warrant further investigation. Whilst the measures of blood oxygen saturation and muscle tissue oxygenation were recorded in this study, we did not measure cerebral oxygenation. Our speculation regarding the greater reduction in exercise during exposure to severe hypoxia is largely supported by a theoretical reduction in brain oxygenation. In comparable levels of hypoxia, others (Amann et al. 2007; Subudhi et al. 2007, 2009; Goodall et al. 2012) have reported such changes, therefore, despite this being a limitation to our findings, it is plausible that comparable levels of cerebral oxygenation may have occurred. We also were able to examine $\mathrm{PETCO}_{2}$ data which closely align with change in cerebral blood flow as previously discussed. In addition, whilst we noted the comparable levels of muscle tissue oxygenation during exercise in hypoxia, further analysis will be required to determine if a stable oxygen condition was met by increases in blood flow or reduced metabolic demand, which may give further insight into the afferent processes that may underlie this observation. Finally, measures of peripheral muscle fatigue would have been useful to quantify the level of fatigue experienced and further support our conclusions regarding peripheral and central mechanisms.

\section{Conclusions}

In conclusion, severe and moderate hypoxia elicited reductions in exercise when controlled at a fixed RPE. The primary cues for determining perceived effort related to the decrease in arterial hypoxemia and increase in ventilation, which was largely driven by an increase in breathing frequency. The strong relationships found between exercise time and both ventilation and $\mathrm{SpO}_{2}$ support the role of these physiological cues in setting the early intensity of perceptually controlled exercise.

Acknowledgements We are very grateful to the participants for their effort and commitment to this study.

Author contributions Conception and design of research (OJ and $\mathrm{MW})$; Acquisition, analysis, and interpretation of data (OJ and $\mathrm{MW}$ ); drafted, edited, and revised manuscript (OJ, MW, and SDP); approved final version of manuscript (OJ, MW, and SDP). All authors agree to be accountable for all the aspects of the work (OJ, MW, and SDP). All authors that qualify for authorship are listed (OJ, MW, and SDP). 


\section{Compliance with ethical standards}

Conflict of interest All authors declare that they have no conflict of interest.

Open Access This article is distributed under the terms of the Creative Commons Attribution 4.0 International License (http://creativeco mmons.org/licenses/by/4.0/), which permits unrestricted use, distribution, and reproduction in any medium, provided you give appropriate credit to the original author(s) and the source, provide a link to the Creative Commons license, and indicate if changes were made.

\section{References}

Amann M, Calbet JAL (2008) Convective oxygen transport and fatigue. J Appl Physiol 104:861-870

Amann M, Eldridge MW, Lovering AT et al (2006a) Arterial oxygenation influences central motor output and exercise performance via effects on peripheral locomotor muscle fatigue in humans. J Physiol 575:937-952

Amann MM, Romer LMLM, Pegelow DFDF et al (2006b) Effects of arterial oxygen content on peripheral locomotor muscle fatigue. J Appl Physiol 101:119-127. https://doi.org/10.1152/japplphysi ol.01596.2005

Amann M, Romer LM, Subudhi AW et al (2007) Severity of arterial hypoxaemia affects the relative contributions of peripheral muscle fatigue to exercise performance in healthy humans. J Physiol 581:389-403

American College of Sports Medicine (2000) ACSM's guidelines for exercise testing and prescription, 6th edn. Lippincott Williams \& Wilkins, Philadelphia

Borg GA (1982) Psychophysical bases of perceived exertion. Med Sci Sports Exerc 14:377-381. https://doi.org/10.1249/00005768198205000-00012

Brick NE, MacIntyre TE, Campbell MJ (2016) Thinking and action: a cognitive perspective on self-regulation during endurance performance. Front Physiol 7:159. https://doi.org/10.3389/fphys .2016 .00159

Brocherie F, Millet GP, Girard O (2017) Psychophysiological responses to repeated-sprint training in normobaric hypoxia and normoxia. Int J Sports Physiol Perform 12:115-123. https://doi.org/10.1123/ ijspp.2016-0052

Cafarelli E, Noble BJ (1976) The effect of inspired carbon dioxide on subjective estimates of exertion during exercise. Ergonomics 19:581-589. https://doi.org/10.1080/00140137608936944

Calbet JA, Boushel R, Rådegran G et al (2003a) Determinants of maximal oxygen uptake in severe acute hypoxia. Am J Physiol Regul Integr Comp Physiol 284:291-303. https://doi.org/10.1152/ajpre gu.00155.2002

Calbet JAL, De Paz JA, Garatachea N et al (2003b) Anaerobic energy provision does not limit Wingate exercise performance in endurance-trained cyclists. J Appl Physiol 94:668-676. https://doi. org/10.1152/japplphysiol.00128.2002

Cohen J (1988) Statistical power analysis for the behavioral sciences, 2nd edn. Erlbaum, Hillsdale, New York

Curtelin D, Morales-Alamo D, Torres-Peralta R et al (2018) Cerebral blood flow, frontal lobe oxygenation and intra-arterial blood pressure during sprint exercise in normoxia and severe acute hypoxia in humans. J Cereb Blood Flow Metab 38:136-150. https://doi. org/10.1177/0271678X17691986

Farra SD, Cheung SS, Thomas SG, Jacobs I (2017) Rate dependent influence of arterial desaturation on self-selected exercise intensity during cycling. PLoS One 12:e0171119. https://doi. org/10.1371/journal.pone.0171119

Ferrari M, Mottola L, Quaresima V (2004) Principles, techniques, and limitations of near infrared spectroscopy. Can J Appl Physiol 29(4):463-487

Fortune JB, Bock D, Kupinski AM et al (1992) Human cerebrovascular response to oxygen and carbon dioxide as determined by internal carotid artery duplex scanning. J Trauma 32:618

Fulco CS, Lewis SF, Frykman PN et al (1996) Muscle fatigue and exhaustion during dynamic leg exercise in normoxia and hypobaric hypoxia. J Appl Physiol 81:1891-1900

Fulco CS, Rock PB, Cymerman A (1998) Maximal and submaximal exercise performance at altitude. Aviat Sp Environ Med 69:793-801

Gandevia SC (2001) Spinal and supraspinal factors in human muscle fatigue. Physiol Rev 81:1725-1789

Gonzalez-Alonso J, Dalsgaard MK, Osada T et al (2004) Brain and central haemodynamics and oxygenation during maximal exercise in humans. J Physiol 557:331-342. https://doi.org/10.1113/jphys iol.2004.060574

Goodall S, González-Alonso J, Ali L et al (2012) Supraspinal fatigue after normoxic and hypoxic exercise in humans. J Physiol 590:2767-2782. https://doi.org/10.1113/jphysiol.2012.228890

Hampson DB, St Clair Gibson A, Lambert MI, Noakes TD (2001) The influence of sensory cues on the perception of exertion during exercise and central regulation of exercise performance. Sport Med 31:935-952. https://doi.org/10.2165/00007256-20013 1130-00004

Hogan MC, Richardson RS, Haseler LJ et al (1999) Human muscle performance and $\mathrm{PCr}$ hydrolysis with varied inspired oxygen fractions: a 31P-MRS study. J Appl Physiol 86:1367-1373

Ide K, Eliasziw M, Poulin MJ (2003) Relationship between middle cerebral artery blood velocity and end-tidal PCO2 in the hypocapnichypercapnic range in humans. J Appl Physiol 95:129-137. https ://doi.org/10.1152/japplphysiol.01186.2002

Jensen JB, Sperling B, Severinghaus JW, Lassen NA (1996) Augmented hypoxic cerebral vasodilation in men during 5 days at 3,810 m altitude. J Appl Physiol 80:1214-1218

Kaufman MP, Forster HV (1996) Reflexes controlling circulatory, ventilatory and airway responses to exercise. Handbook of physiology, exercise: regulation and integration of multiple systems, 29th edn. Oxford University Press, New York

Killian KJ (1998) Sense of effort and dyspnoea. Monaldi Arch chest Dis Arch Monaldi per le Mal del torace 53:654-660

McMorris T, Hale BJ, Barwood M et al (2017) Effect of acute hypoxia on cognition: a systematic review and meta-regression analysis. Neurosci Biobehav Rev 74:225-232. https://doi.org/10.1016/j. neubiorev.2017.01.019

Millet GY, Muthalib M, Jubeau M et al (2012) Severe hypoxia affects exercise performance independently of afferent feedback and peripheral fatigue. J Appl Physiol 112:1335-1344. https://doi. org/10.1152/japplphysiol.00804.2011

Morgan WP (1994) Psychological components of effort sense. Med Sci Sports Exerc 26:1071-1077

Nattie E, Li A (2009) Central chemoreception is a complex system function that involves multiple brain stem sites. J Appl Physiol 106:1464-1466. https://doi.org/10.1152/japplphysiol.00112.2008

Neubauer JA, Melton JE, Edelman NH (1990) Modulation of respiration during brain hypoxia. J Appl Physiol 68:441-451. https://doi. org/10.1152/jappl.1990.68.2.441

Nicolo A, Marcora SM, Sacchetti M (2016) Respiratory frequency is strongly associated with perceived exertion during time trials of different duration. J Sports Sci 34:1199-1206. https://doi. org/10.1080/02640414.2015.1102315

Niedermeier M, Weisleitner A, Lamm C et al (2017) Is decision making in hypoxia affected by pre-acclimatisation? A randomized 
controlled trial. Physiol Behav 173:236-242. https://doi. org/10.1016/j.physbeh.2017.02.018

Noakes T (2004) Linear relationship between the perception of effort and the duration of constant load exercise that remains. J Appl Physiol 96:1571-1572. https://doi.org/10.1152/japplphysi ol.01124.2003 1572-1573.

Poulin MJ, Fatemian M, Tansley JG et al (2002) Changes in cerebral blood flow during and after $48 \mathrm{~h}$ of both isocapnic and poikilocapnic hypoxia in humans. Exp Physiol 87:633-642

Ringelstein EB, Van Eyck S, Mertens I (1992) Evaluation of cerebral vasomotor reactivity by various vasodilating stimuli: comparison of CO2 to acetazolamide. J Cereb Blood Flow Metab 12:162-168. https://doi.org/10.1038/jcbfm.1992.20

Robertson RJ (1982) Central signals of perceived exertion during dynamic exercise. Med Sci Sports Exerc 14:390-396

Romer LM, Haverkamp HC, Lovering AT et al (2006) Evidence of neuromuscular fatigue after prolonged cycling exercise. Am J Physiol Regul Integr Comp Physiol 290:365-375. https://doi.org/10.1152/ ajpregu.00332.2005

Romer LM, Haverkamp HC, Amann M et al (2007) Effect of acute severe hypoxia on peripheral fatigue and endurance capacity in healthy humans. Am J Physiol Regul Integr Comp Physiol 292:R598-R606. https://doi.org/10.1152/ajpregu.00269.2006

St Clair Gibson A, Lambert EV, Rauch LHG et al (2006) The role of information processing between the brain and peripheral physiological systems in pacing and perception of effort. Sports Med 36:705-722

Subudhi AW, Dimmen AC, Roach RC (2007) Effects of acute hypoxia on cerebral and muscle oxygenation during incremental exercise. J Appl Physiol 103:177-183. https://doi.org/10.1152/japplphysi ol.01460.2006

Subudhi AW, Miramon BR, Granger ME, Roach RC (2009) Frontal and motor cortex oxygenation during maximal exercise in normoxia and hypoxia. J Appl Physiol 106:1153-1158

Tipton MJ, Harper A, Paton JFR, Costello JT (2017) The human ventilatory response to stress: rate or depth? J Physiol 595:5729-5752. https://doi.org/10.1113/JP274596
Torres-Peralta R, Losa-Reyna J, Morales-Alamo D et al (2016) Increased PIO2 at exhaustion in hypoxia enhances muscle activation and swiftly relieves fatigue: a placebo or a PIO2 dependent effect? Front Physiol 7:333. https://doi.org/10.3389/fphys .2016 .00333

Tucker R (2009) The anticipatory regulation of performance: the physiological basis for pacing strategies and the development of a perception-based model for exercise performance. Br J Sports Med 43:392-400. https://doi.org/10.1136/bjsm.2008.050799

Tucker R, Marle T, Lambert EV, Noakes TD (2006) The rate of heat storage mediates an anticipatory reduction in exercise intensity during cycling at a fixed rating of perceived exertion. J Physiol 574:905-915. https://doi.org/10.1113/jphysiol.2005.101733

Urbaniak GC, Plous S (2015) Research randomizer (version 4.0) [computer software]. http://www.randomizer.org/

Venhorst A, Micklewright D, Noakes TD (2018) Towards a threedimensional framework of centrally regulated and goal-directed exercise behaviour: a narrative review. Br J Sports Med 52:957966. https://doi.org/10.1136/bjsports-2016-096907

Vogiatzis I, Louvaris Z, Habazettl H et al (2011) Frontal cerebral cortex blood flow, oxygen delivery and oxygenation during normoxic and hypoxic exercise in athletes. J Physiol 589:4027-4039. https://doi. org/10.1113/jphysiol.2011.210880

Vovk A, Cunningham DA, Kowalchuk JM et al (2002) Cerebral blood flow responses to changes in oxygen and carbon dioxide in humans. Can J Physiol Pharmacol 80:819-827

Publisher's Note Springer Nature remains neutral with regard to jurisdictional claims in published maps and institutional affiliations. 